\title{
Rekonstruktionsoptimierung und Leistungsfähigkeit eines neurochirurgisch eingesetzten intraoperativen Flat-Panel-Systems
}

\author{
Stefan Röhrer, Ralph König, Christian Rainer Wirtz, Peter Richter, Florian Gebhard, \\ Thomas Kapapa
}

\begin{abstract}
Zusammenfassung
Die digitale Subtraktionsangiografie stellt den Goldstandard in der vaskulären Neurochirurgie dar. Durch die Anwendung einer intraoperativen Angiografie, welche durch den Hybridoperationssaal mit dem System Artis zeego möglich ist, kann diese Kontrolle noch während der Operation, bei geöffnetem Schädel durchgeführt werden. Unterschiedliche 2-D- und 3-D-Darstellungsmöglichkeiten der akquirierten Bilddaten machen es dem Operateur möglich, die operative Strategie den daraus resultierenden Befunden ggf. anzupassen.
\end{abstract}

\section{The Efficiency of a Neurosurgical Used Intraoperative Flat-Panel- System}

The digital subtraction angiography is the method of choice to control the results after neurovascular surgery. Intraoperative angiography performed in a hybrid-operation environment allows you to change surgical strategies immediately after image acquisition. Different 2D- and 3D-visualisations of the intraoperative acquired datasets guide the assessment of the surgical result.

\section{Einleitung}

Die intraoperative Angiografie (IOA) wurde während der 60er-Jahre in die Neurochirurgie eingeführt. Die Hauptindikation war zunächst die Verbesserung der mikrochirurgischen Versorgung intrakranieller Aneurysmen.

Der Nutzen der IOA in der Aneurysmachirurgie liegt v. a. darin, einen Aneurysmarest einerseits, den Verschluss oder die relevante Einengung benachbarter Gefäße andererseits, auszuschließen.

5-7,3\% aller mikrochirurgisch versorgter Aneurysmen sind inkomplett verschlossen. Als Konsequenz besteht ein anhaltendes Risiko für eine Ruptur, weitere Behandlungen können erforderlich werden [4]. In etwa 3-9\% kann es zu einem Verschluss oder einer relevanten Einengung benachbarter Gefäße mit der Gefahr eines Hirninfarkts und bleibenden neurologischen Defiziten kommen $[2,8]$.

OP-JOURNAL 2014; 30: 164-168

(c) Georg Thieme Verlag KG Stuttgart · New York DOI http://dx.doi.org/10.1055/s-0034-1383301
Weitere Indikationen für die IOA sind die mikrochirurgische Versorgung arteriovenöser Malformationen (AVM) und duraler AV-Fisteln (dAVF). Ein residueller AVM-Nidus respektive ein persistierender AV-Shunt mit der Folge eines persistierenden Blutungsrisikos kann in bis zu 8,9 bzw. $18,8 \%$ nach chirurgischer Resektion nachgewiesen werden [1].

Trotz des nachgewiesenen Nutzens der IOA in der Versorgung neurovaskulärer Krankheitsbilder konnte nie ein Konsensus im Hinblick auf ihre routinemäßige Nutzung erzielt werden. Die IOA konnte sich in der chirurgischen Behandlung neurovaskulärer Erkrankungen nie flächendeckend durchsetzen. Die Gründe hierfür sind vielfältig, um nur die wichtigsten zu nennen: Die zunächst meist C-Bogen-basierte IOA war aufwendig und in ihrer Qualität begrenzt. Darüber hinaus war die Komplikationsrate (Embolien, Gefäßverletzungen, Dissektionen) der IOA relativ hoch [3].

Mit der zunehmenden Realisierung des Konzepts des Hybrid-OPs werden hochleistungsfähige High-End-Bildgebungssysteme in die chirurgische Umgebung integriert. Neben der intraoperativen Kernspintomografie [5] und dem intraoperativen CT [9] werden vor allem moderne, vielfältig einsetzbare Flat-PanelBildgebungssysteme verwendet. Der Einsatz moderner Flat-Panel-Technologie im OP bietet auch für die Behandlung neurovaskulärer Erkrankungen, neben der reinen IOA, fortgeschrittene Möglichkeiten der intraoperativen Bildgebung [7].

Ziel des Übersichtsartikels ist die Darstellung der Möglichkeiten der intraoperativen neurovaskulären Bildgebung in einem auf multidisziplinäre Nutzung ausgerichteten Hybridoperationssaal mit dem System Artis zeego (Siemens Healthcare, Deutschland) darzulegen.

\section{Aufbau des Hybridoperationssaals}

Kernstücke des interdisziplinär genutzten Hybridoperationssaals sind der Artis zeego (Siemens Healthcare, Deutschland) und der TruSystem 7500 Operationstisch (Trumpf, Deutschland, Abb. 1). Durch die Kopplung beider Elemente ist prinzipiell eine präzise abgestimmte Bewegungssteuerung zwischen den beiden Komponenten möglich. Allerdings werden Anbauteile, wie die für neurochirurgische Eingriffe erforderliche Kopfklemme, vom System derzeit noch nicht erkannt, sodass für die kraniale Bildgebung derzeit noch eine visuelle Kollisionsüberwachung zwischen Tisch und Zeego erforderlich ist. Durch die Größe der Flat-Panel-Detektoren $(30 \times 40 \mathrm{~cm})$ ist die Darstellung des gesamten Schädels möglich. Die Flexibilität und Bewegungsgeschwindigkeit des Artis zeego, eines auf einem 6-Achsen-Robotersystem aufgebauten Flat-Panel-Detektors, ermöglicht verschiedene Modalitäten der 2-D-, 3-D- und physiologischen vaskulären Bildgebung:

- digitale Subtraktionsangiografie mit extrem hohen Bildraten

- 3-D-Rotationsangiografie

- Dyna-CT mit CT-ähnlichem Weichteilkontrast 


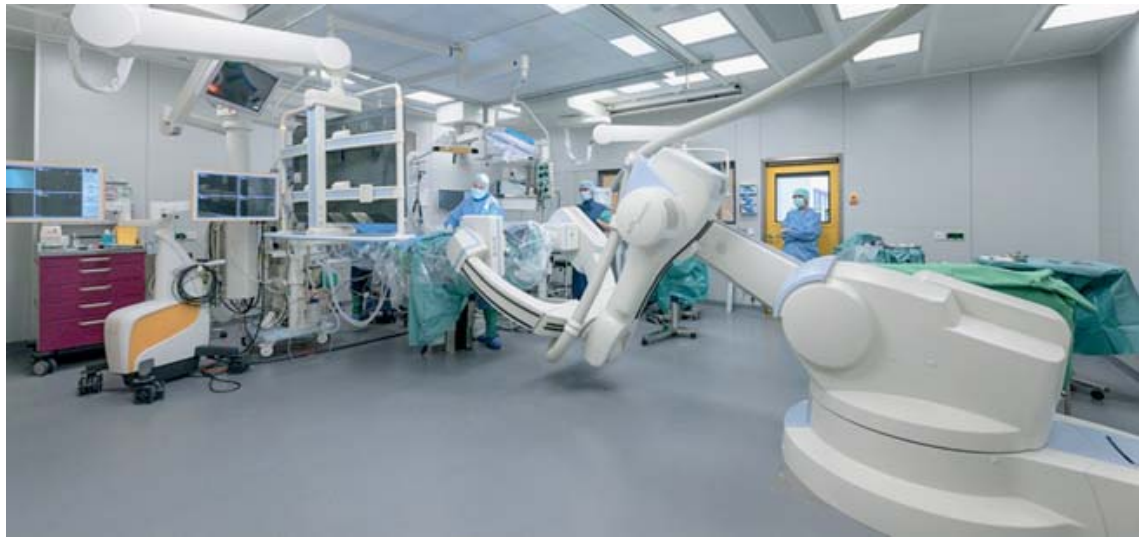

Abb. 1 Aufbau des Hybridoperationssaals mit dem System Artis zeego und integriertem Operationstisch.

- physiologische Bildgebung (zerebrales Blutvolumen, CBV)

Roadmap- oder Overlay-Techniken ermöglichen die sichere Katheterführung für die selektive zerebrale Gefäßdiagnostik.

\section{Leistungsfähigkeit und Grenzen des intraoperativen Bildgebungssystems}

\section{Digitale Subtraktionsangiografie}

Die Möglichkeiten und die Qualität der digitalen Subtraktionsangiografie sind mit der neurovaskulären Standarddiagnostik annähernd vergleichbar. Insbesondere zum Nachweis eines residuellen AV-Shunts bei der Resektionskontrolle von AVMs und dAVFs ermöglicht das System extrem hohe Bildraten. Durch Automap-Technik kann selbst bei extredrehter Kopf) anhand zuvor akquirierter 3-D-Bilddaten saubere a.-p.- und laterale Projektionsaufnahmen aufgenommen werden (Abb. 2).

\section{Selektive intraarterielle 3-D-Angiografie und Dyna-CT}

Der Artis zeego ist ein aufgrund seiner hohen Rotationsgeschwindigkeit extrem leistungsfähiges 3-D-Bildgebungssystem. Für die intraoperative Kontrolle von zerebralen Aneurysmen ist insbesondere die sog. Dual-Volume-Rekonstruktion wertvoll. Dabei werden 2 3-D-Bilddatenmen Operationslagerungen (stark ge-

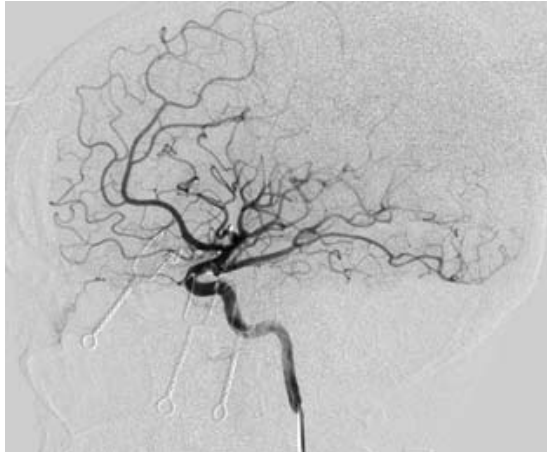

Abb. 2 Intraoperative Angiografie der A. carotis interna mit Darstellung der $A$. cerebri media als aneurysmatragendes Gefäß (Pfeil).

sätze (Maske, d.h. knöcherner Schädel und Implantate [Coils oder Clips] und der Angiografiedatensatz) separat voneinander rekonstruiert und bearbeitet und abschließend fusioniert. Jedes Volumen kann getrennt weiter modifiziert, z.B. gefenstert werden (Abb. 3 und 4). Diese Art der 3-D-Darstellung ermöglicht eine rundum 3-D-Ansicht des Gefäßbaums und Aneurysmaclips. Somit ist es möglich, die im intraoperativen Situs nicht einsehbare Rückseite des Gefäßes darzustellen (Abb. 5, 6 und 7). Dies ist insbesondere bei proximalen Aneurysmen, bspw. der A. carotis, bedeutsam. Durch die 3-D-Rekonstruktion kann ein Restaneurysma bzw. eine durch den Clip verursachte Gefäßstenose sofort detektiert und die Clipposition ggf. verändert

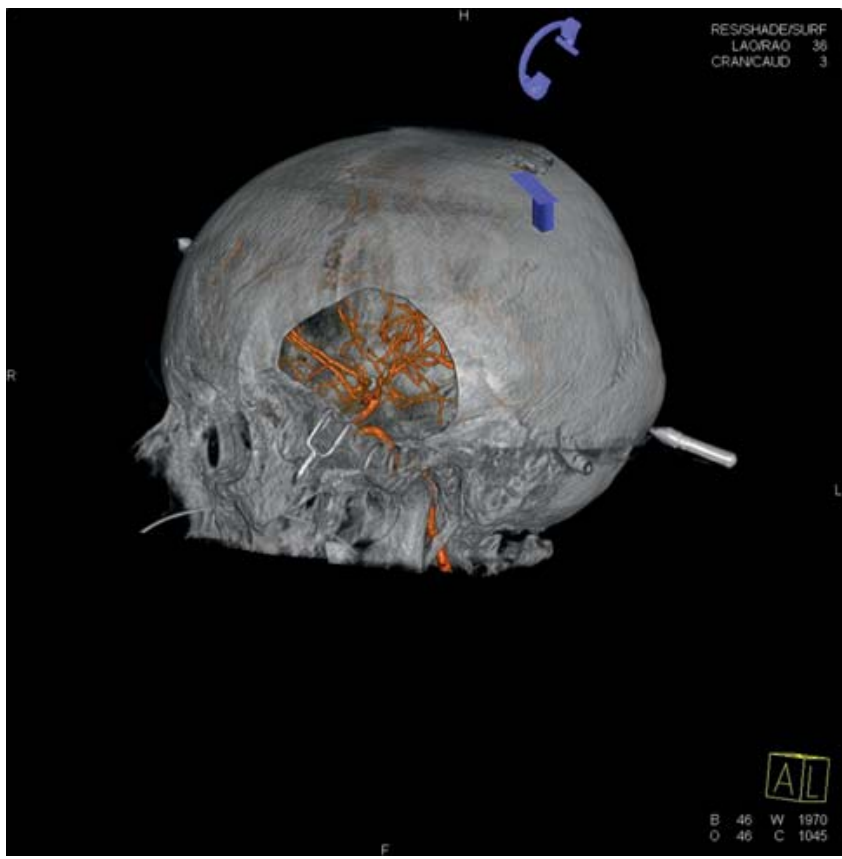

Abb. 3 Rekonstruktion zur Darstellung des knöchernen Schädels, des arteriellen Gefäßbaums und des Aneurysmaclips.

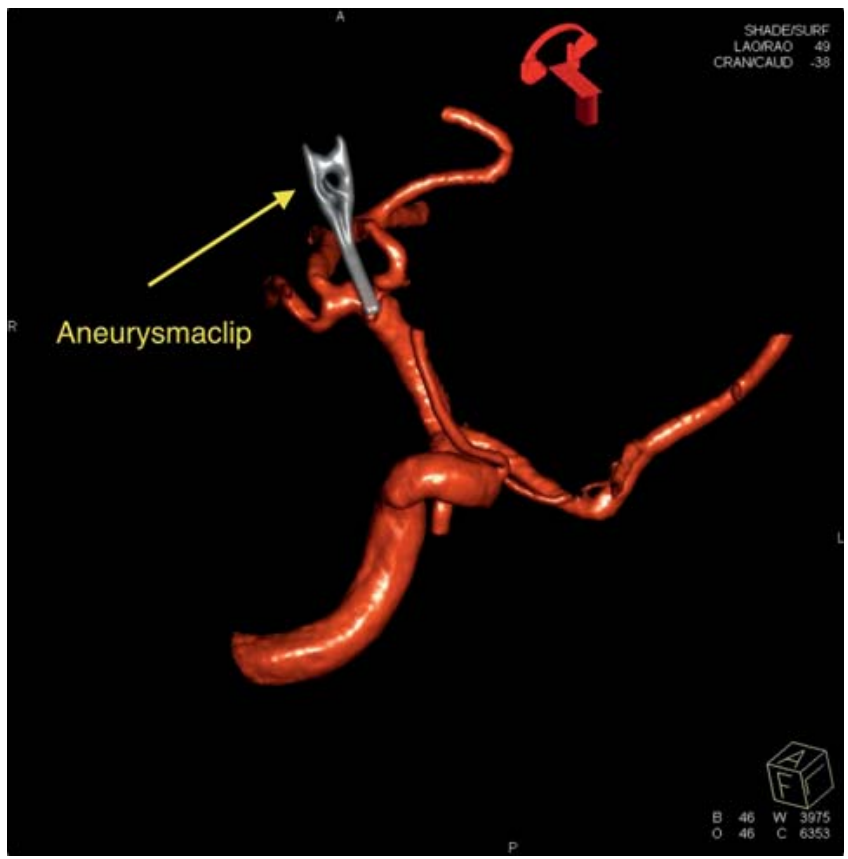

Abb. 4 3-D-Rekonstruktion mit platziertem Aneurysmaclip. 


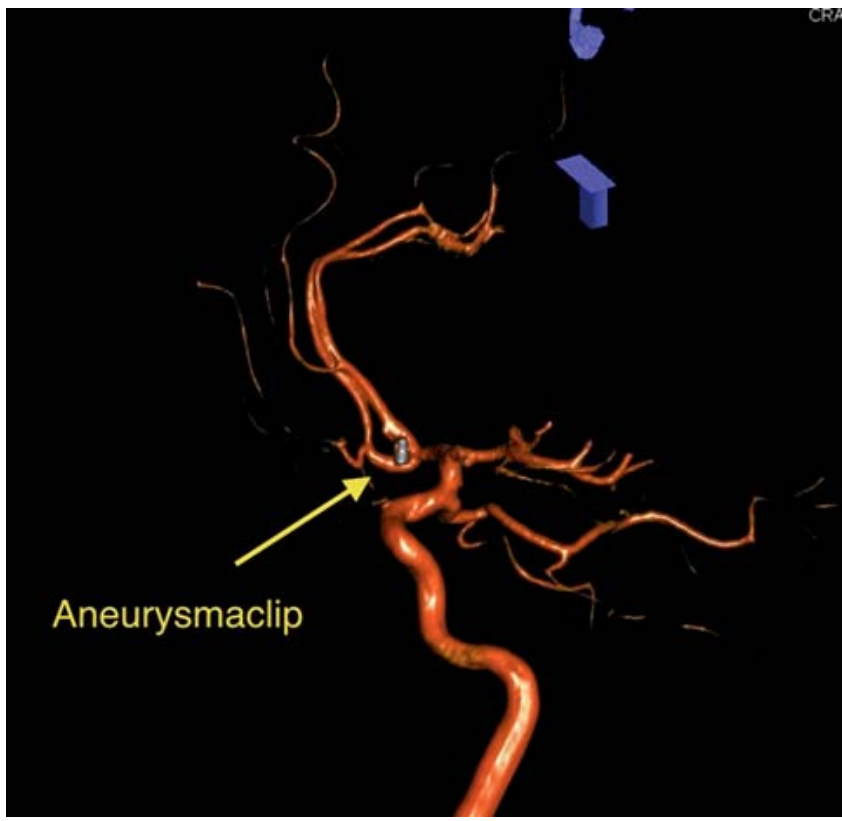

Abb. 5 3-D-Rekonstruktion des arteriellen Gefäßbaums nach Setzen des Aneurysmaclips ohne Nachweis eines Restaneurysmas.

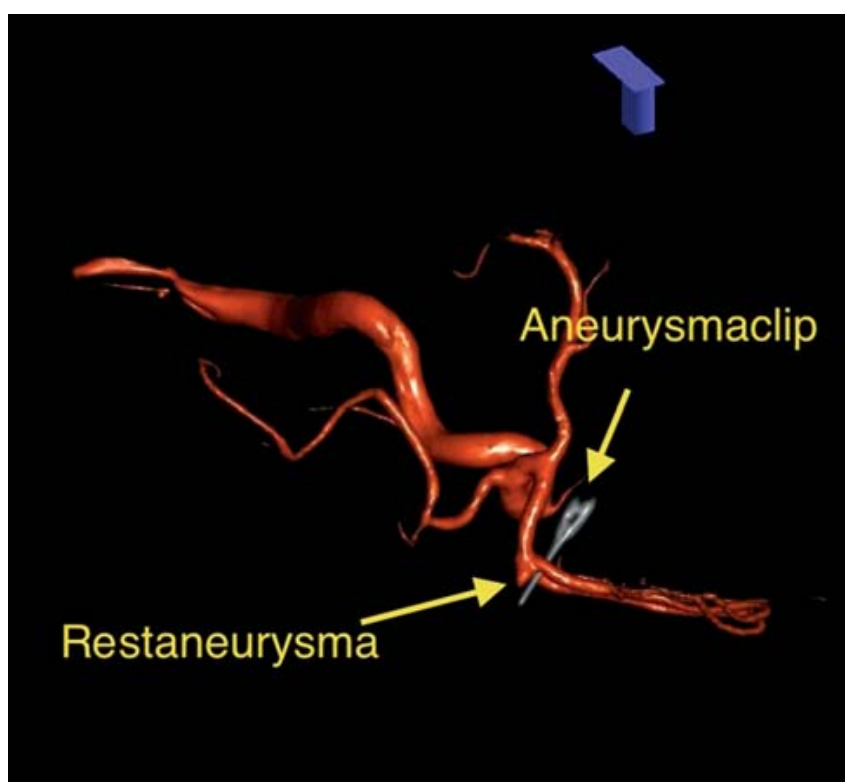

Abb. 7 Darstellung des Restaneurysmas.

werden, um neurologische Folgeschäden zu verhindern.

\section{3-D-Rotationsangiografie mit intravenös verabreichtem Kontrastmittel}

Insbesondere bei Patienten mit erhöhtem Risikoprofil für eine intraoperative selektive intraarterielle Angiografie (z.B. ausgeprägte pAVK, Karotisstenose) kann eine intravenöse Kontrastmittelapplikation erfolgen. Die sog. i.v. 3-D-Rotations-

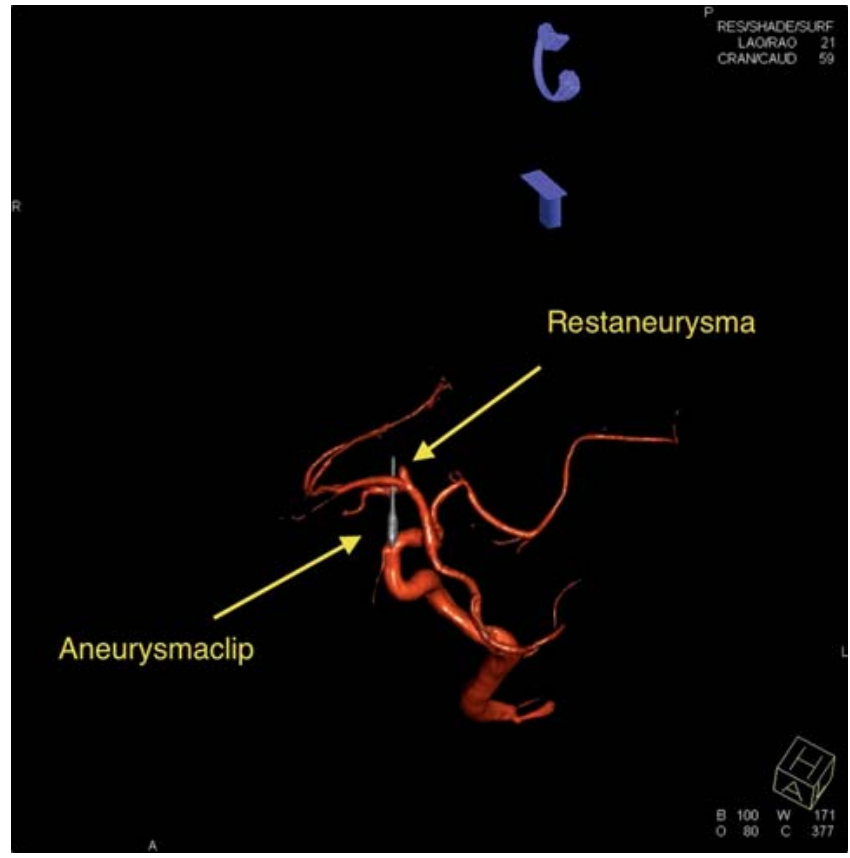

Abb. 6 Nach Rotation des Bilddatensatzes zeigt sich an der vom Operateur abgewandten Seite ein Restaneurysma.

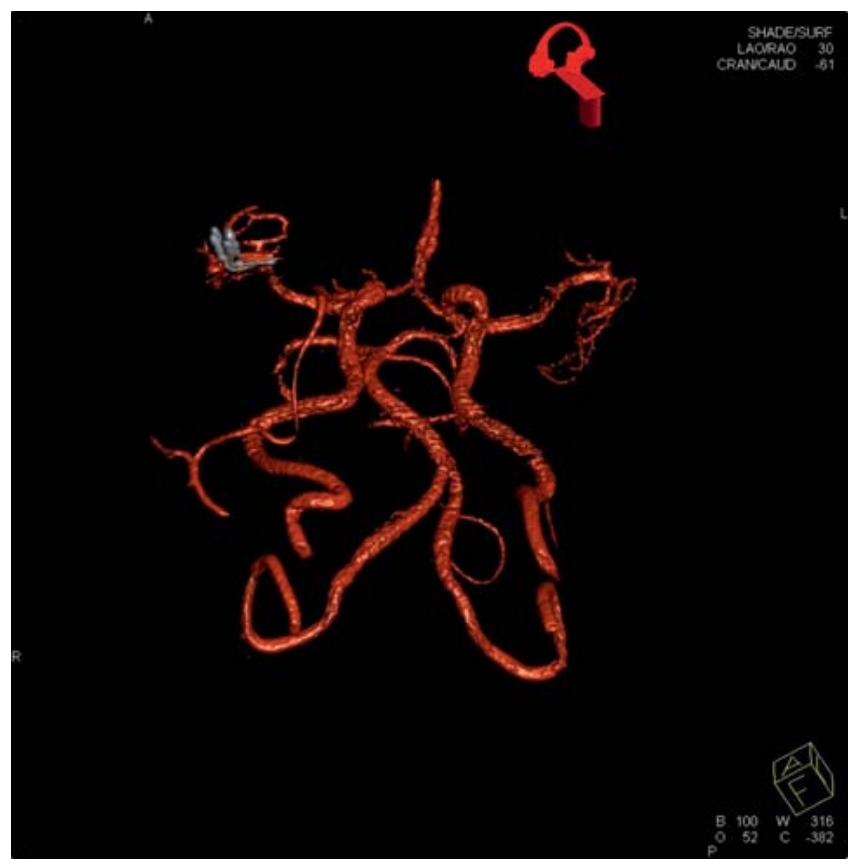

Abb. 8 3-D-Rekonstruktion des arteriellen Gefäßbaums nach i.v. Kontrastmittelgabe.

angiografie wird hinsichtlich ihrer Leistungsfähigkeit derzeit erprobt und verfeinert. Sie bietet die Möglichkeit einer risikoarmen Gefäßbildgebung, muss aber im Hinblick auf ihre Bildqualität und deren Einflussvariablen noch verbessert und standardisiert werden (Abb. 8).
Grenzen der intraoperativen Bildgebung mit dem Flat-Panel-System

Die Grenzen der Bildgebung ergeben sich einerseits durch Artefakte, welche durch die spezielle intraoperative Anwendungen bedingt sind: Kopfhalterung, Fixierungspins, Aufhärtungsartefakte an der Grenzzone zwischen Luft und OP-Situs (Abb. 9, 10, 11, 12 und 13). Beispielswei- 


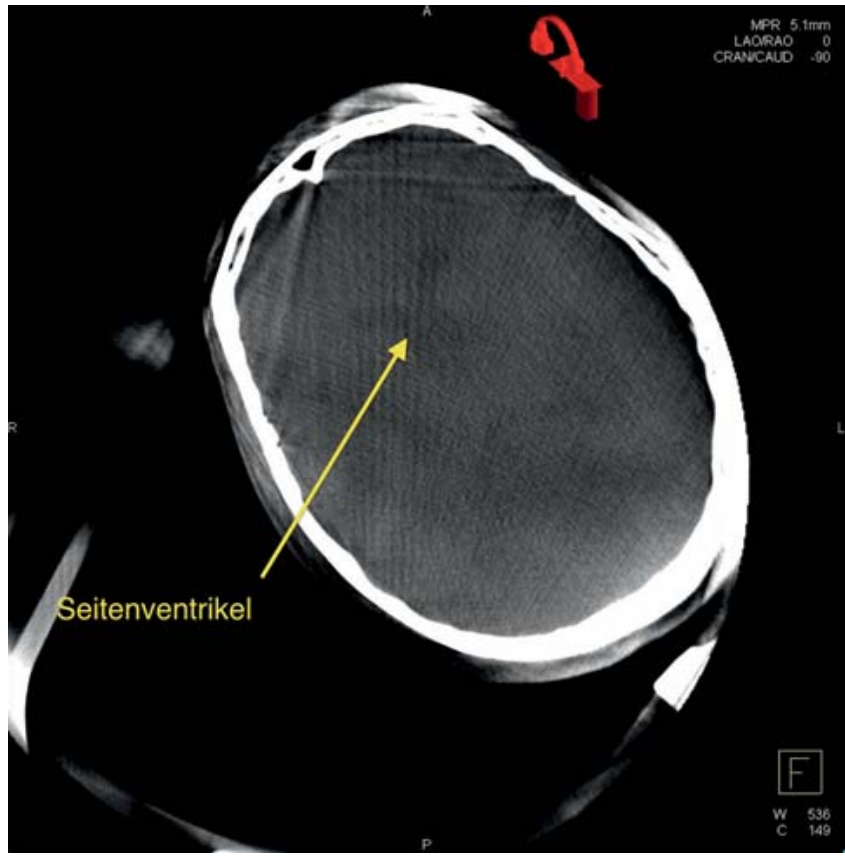

Abb.9 Parenchymdarstellung mittels Rotationsbildgebung.

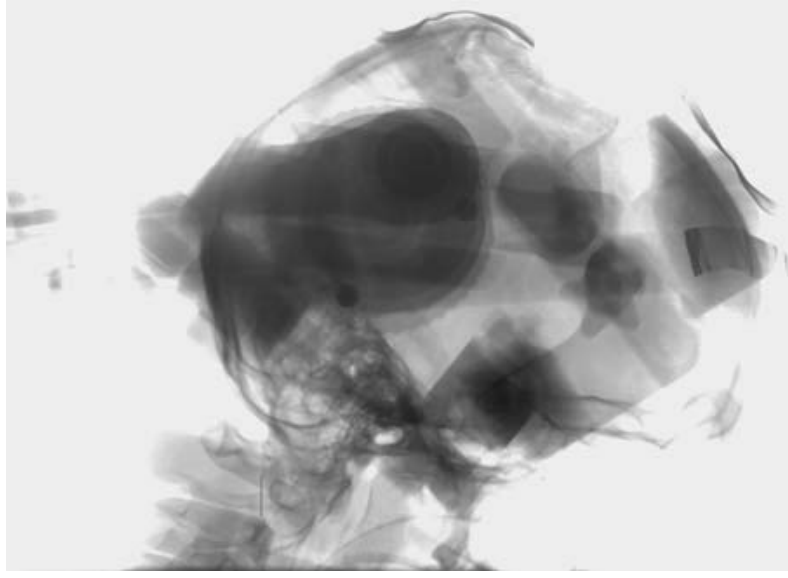

Abb. 11 Artefaktüberlagerte Rotationsbildgebung.

se konnten durch die Erprobung unterschiedlicher Materialien von verschiedenen Herstellern inzwischen Fixierungspins mit deutlich reduzierter Artefaktbildung angeboten werden. Insbesondere Aufhärtungsartefakte zwischen Luft und OP-Situs können die Bildqualität der IOA einschränken, weshalb bei der Vorbereitung zur Bildgebung darauf geachtet werden sollte, den Situs mit steriler Spüllösung aufzufüllen.

Insbesondere die für die i.v. Bildakquisition erforderliche 10-Sekunden-Rotation erweist sich als stärker artefaktempfindlich als die vergleichbare 6-SekundenRotation der intraarteriellen Rotationsangiografie. Weitere Einschränkungen der 3-D-Nachbearbeitung ergeben sich

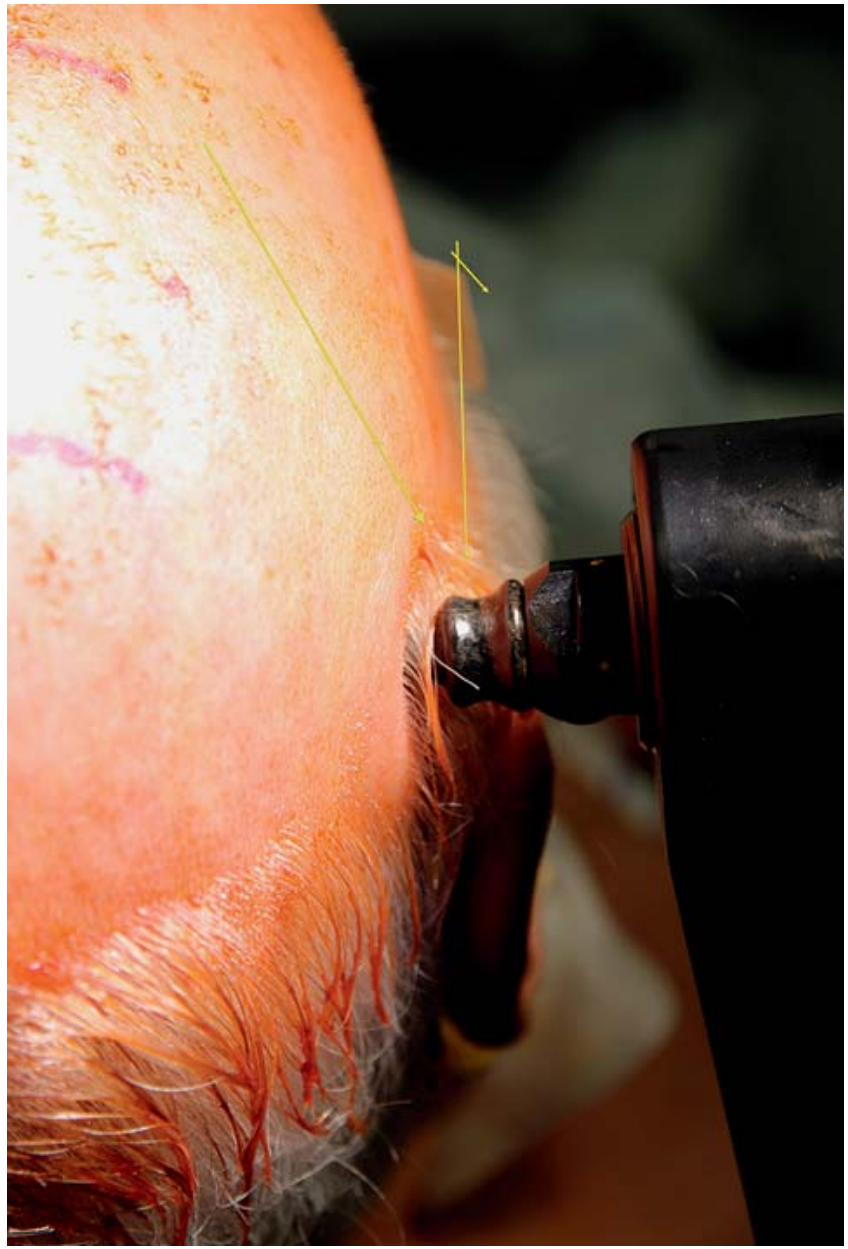

Abb. 10 Metallpin zur Fixierung des Schädels während der Operation.

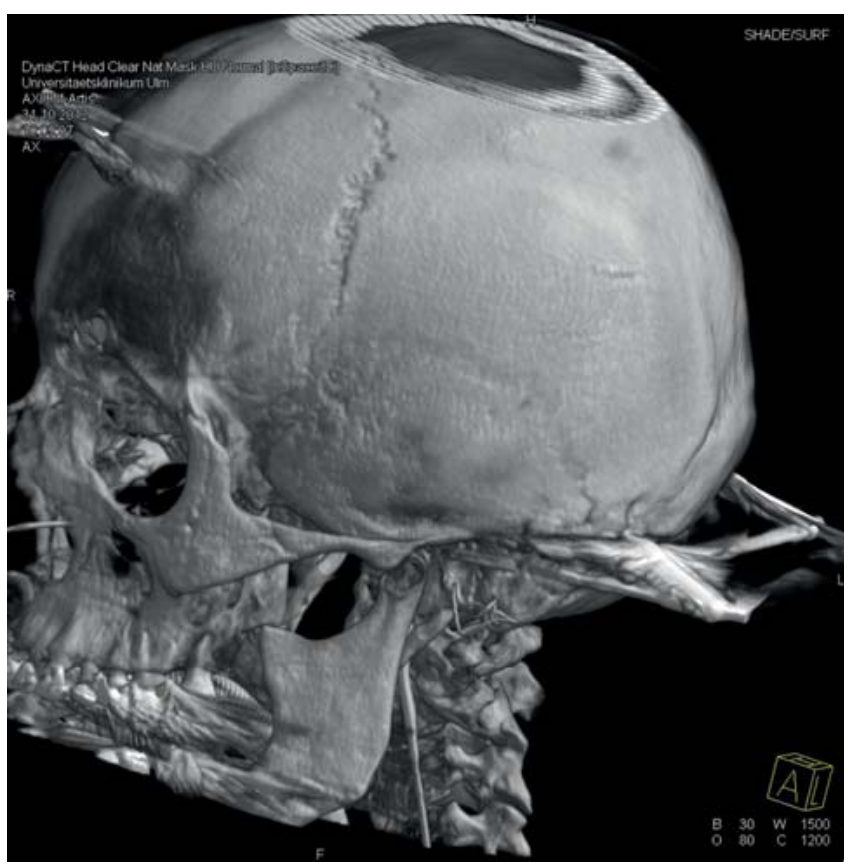

Abb. 12 3-D-Rekonstruktion der Schädelkalotte mit Pinartefakten. 


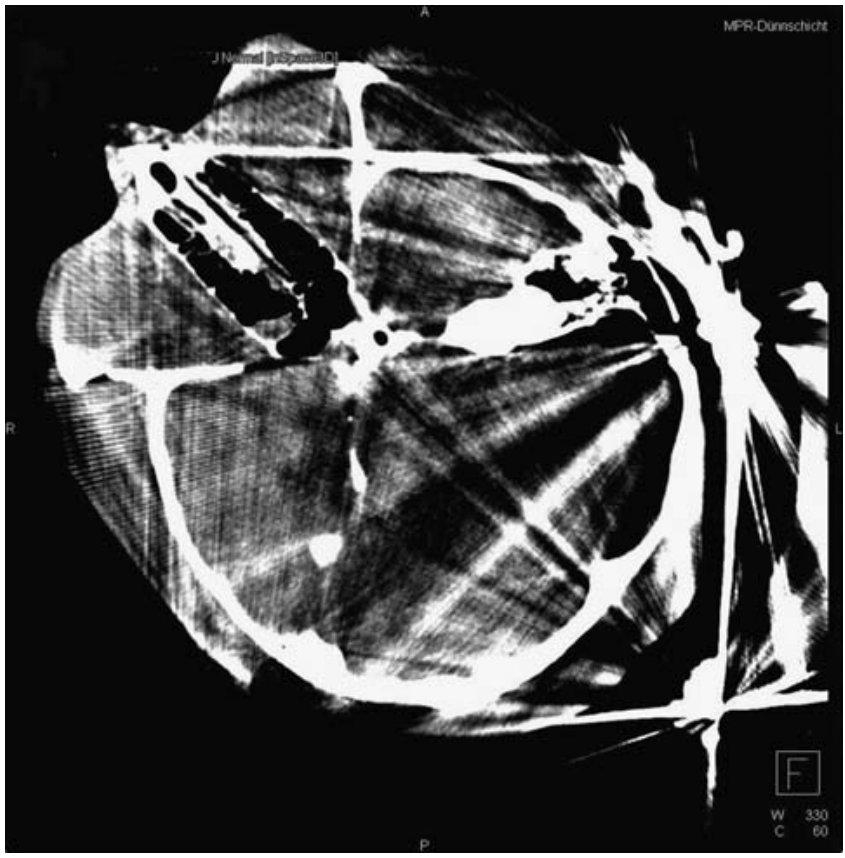

Abb. 13 Artefaktüberlagerte, axiale Dyna-CT-Bildgebung.

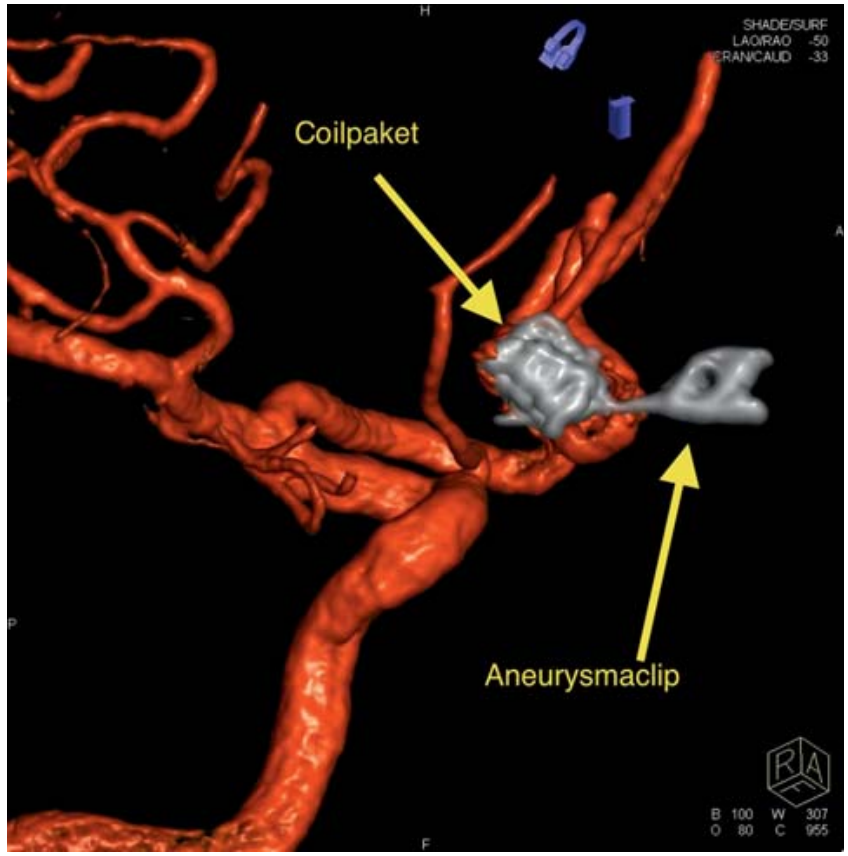

Abb. 14 Primär gecoiltes Aneurysma nach Positionierung des Clips. bei reperfundierten endovaskulär versorgten Aneurysmen, bei welchen ein sekundäres Clipping notwendig ist. Durch die in das Aneurysma eingebrachten Platincoilschlingen ist eine Darstellung des Aneurysmahalses bildmorphologisch deutlich erschwert (Abb. 14)

\section{Schlussfolgerung}

Die intraoperative Anwendung des Systems Artis zeego in der vaskulären Neurochirurgie ermöglicht eine unmittelbare Kontrolle des Operationsziels während des Eingriffs. Durch die hohe Bildgebungsqualität kann eine den Patienten weiter belastende Kontrollangiografie im postoperativen Verlauf vermieden werden.

Roadmap- und Overlay-Techniken ermöglichen insbesondere die sichere selektive Sondierung der hirnversorgenden Gefäße, wodurch die immer wieder berichtete hohe Komplikationsrate der IOA sicher reduziert werden kann. Diesen Vorteilen stehen die hohen Kosten eines solchen Hybridoperationssaals entgegen. Um eine Rentabilität zu erreichen, bedarf es einer vollen Ausnutzung des Systems und der damit verbundenen personellen und technischen Ressourcen. Daher bietet sich ein multidisziplinärer Ansatz an, bei welchem der Hybridoperationssaal von unterschiedlichen Fachabteilungen (Neurochirurgie,
Traumatologie, Herzchirurgie, Gefäßchirurgie und HNO) genutzt wird, um die Ressourcen optimal einzusetzen.

Für kleinere Kliniken ohne maximalversorgenden Ansatz ist die Anschaffung eines Systems dieses Ausmaßes aufgrund der Kosten, des personellen Aufwands sowie einer hohen personellen Lernkurve kritisch zu diskutieren.

Hierbei muss bedacht werden, dass es sich um ein sehr komplexes Gesamtsystem handelt, dessen Möglichkeiten nicht durch eine einmalige Einweisung ausgeschöpft werden können.

An unserer Klinik zeigten sich durch eine hohe Auslastung des Hybridoperationsaals eine rasche Optimierung der Arbeitsabläufe sowie eine Etablierung der unterschiedlichen bildgebenden Protokolle, welche letztlich der Patientensicherheit dienen und somit den größten Vorteil dieses Systems widerspiegeln.

\section{Literatur}

${ }^{1}$ Chalouhi N, Theofanis T, Jabbour P et al. Safety and efficacy of intraoperative angiography in craniotomies for cerebral aneurysms and arteriovenous malformations: a review of 1093 consecutive cases. Neurosurgery 2012; 71: 1162-1169

${ }^{2}$ Chiang VL, Gailloud P, Murphy KJ et al. Routine intraoperative angiography during aneurysm surgery. J Neurosurg 2002; 96: 988-992
${ }^{3}$ Friedman JA, Kumar R. Intraoperative angiography should be standard in cerebral aneurysm surgery. BMC Surgery 2009; 9: 7

${ }^{4}$ Hauck EF, Wohlfeld B, Welch BG et al. Clipping of very large or giant unruptured intracranial aneurysms in the anterior circulation: an outcome study. J Neurosurg 2008; 109: 1012-1018

${ }^{5}$ Hlavac M, König R, Halatsch $M$ et al. [Intraoperative magnetic resonance imaging. Fifteen years' experience in the neurosurgical hybrid operating suite]. Unfallchirurg 2012; 115 : 121-124

${ }^{6}$ Kivisaari RP, Porras M, Ohman J et al. Routine cerebral angiography after surgery for saccular aneurysms: is it worth it? Neurosurgery 2004; 55: 1015-1024

7 Schaller K, Kotowski M, Pereira $V$ et al. From intraoperative angiography to advanced intraoperative imaging: the geneva experience. Acta Neurochir Suppl 2011; 109: 111-115

${ }^{8}$ Tang G, Cawley CM, Dion JE et al. Intraoperative angiography during aneurysm surgery: a prospective evaluation of efficacy. J Neurosurg 2002; 96: 993-999

${ }^{9}$ Uhl E, Zausinger S, Morhard D et al. Intraoperative computed tomography with integrated navigation system in a multidisciplinary operating suite. Neurosurgery 2009; 64 (5 Suppl. 2): S231-S239

\section{Stefan Röhrer}

Assistenzarzt

Klinik für Neurochirurgie

Universitätsklinikum Ulm

Albert-Einstein-Allee 23

89081 Ulm

stefan.roehrer@uniklinik-ulm.de 